Paidéia, 2005, 15(30), 11-20

\title{
A CIÊNCIA DO DESENVOLVIMENTO HUMANO: AJUSTANDO O FOCO DE ANÁLISE ${ }^{1}$
}

\author{
Maria Auxiliadora Dessen² \\ Universidade de Brasília \\ Miriam Teresa Domingues Guedea \\ Universidade de Sonora - México
}

\begin{abstract}
Resumo: Os avanços ocorridos nas últimas décadas, presentes nos domínios interdisciplinares da ciência do desenvolvimento, propõem uma mudança na forma de pensar o estudo do desenvolvimento humano, tendo um forte impacto na pesquisa. Neste artigo, são discutidos princípios e conceitos básicos que têm, recentemente, norteado a definição do próprio conceito de desenvolvimento humano, dentre eles, estrutura, temporalidade, mudanças e continuidades. Destaca-se, também, a necessidade de adotar, na prática da pesquisa, uma visão sistêmica do processo de desenvolvimento, implicando em análises de sistemas complexos e integrados em todos os seus níveis: genético, neural, comportamental e ambiental (físico, social e cultural), interagindo ao longo do tempo e traçando trajetórias de caráter probabilístico.
\end{abstract}

Palavras-chave: ciência do desenvolvimento humano; pesquisa interdisciplinar; fatores biológicos e culturais, ciclo de vida.

\section{DEVELOPMENTAL SCIENCE: RETHINKING THE FOCUS OF ANALYSIS}

Abstract: The interdisciplinary field of developmental science showed a significant progress in the last decades. This progress has changed the view on the ways of thinking about the study of human development, having a strong impact in the research designs. This article aims to discuss basic concepts and principles that recently guide the definition of the human development concept such as structure, temporality, changes and continuities. It is also emphasized the necessity of adopting a systemic approach to study the developmental process. It means that it is important to analyze the complex and integrated systems considering the genetic, neural, behavioral, and environmental (physical, social, and cultural) levels interacting across time and forming probabilistic trajectories of development.

Key-words: developmental science; interdisciplinary research; cultural and biological factors; life course.

\section{Desenvolvimento Humano: Ajustando o Foco de Análise}

A ciência do desenvolvimento refere-se ao conjunto de estudos interdisciplinares que se dedicam a entender os fenômenos relacionados com o desenvolvimento humano, englobando as áreas social, psicológica e biocomportamentais (Magnusson \& Cairns, 1996). Como tal, a ciência do desenvolvimento foca-

\footnotetext{
${ }^{1}$ Artigo recebido para publicação em 20/09/2004; aceito em 23/12/ 2004.

${ }^{2}$ Endereço para correspondência: Maria Auxiliadora Dessen, ColinaUnB, Bloco E, Apto 205, Campus da Universidade de Brasília-UnB
} Brasília-DF, CEP: 70904-105, E-mail: dessen@unb.br liza a ontogênese dos processos evolutivos, destacando as trajetórias no ciclo de vida do indivíduo, considerando-o como ser biológico inserido em determinado tempo e espaço, o que implica enfatizar as mudanças biológicas, temporais, culturais e sociais. O seu foco de análise varia desde os eventos genéticos até os processos culturais, desde os fisiológicos até as interações sociais, com os padrões de adaptação sendo entendidos mediante interações dos níveis internos e externos ao indivíduo.

Este artigo tem como objetivo discutir alguns conceitos em psicologia do desenvolvimento humano, considerando os avanços ocorridos nas últimas décadas, presentes nos domínios interdisciplinares da 


\section{Maria Auxiliadora Dessen}

ciência do desenvolvimento, que propõe uma mudança na forma de pensar o estudo do desenvolvimento humano (Aspesi, Dessen \& Chagas, no prelo). Na primeira parte, discute-se o próprio conceito de desenvolvimento, com destaque para as noções de estrutura, temporalidade, mudança, padrões de mudança e critérios intelectuais e sociais para distinguir as mudanças associadas ao desenvolvimento. Na segunda parte, descrevem-se os conceitos de continuidade e mudança e ressalta-se a necessidade de se adotar a abordagem do curso de vida para estudar os processos de desenvolvimento humano. A terceira parte é dedicada a enfatizar a importância de que as pesquisas levem em conta as inter-relações entre os aspectos biológicos, sociais, culturais e históricos. $\mathrm{Na}$ quarta parte, destaca-se a influência da cultura no desenvolvimento e, finalmente, são tecidas algumas considerações a respeito da importância de se adotar conceitos e pressupostos interdisciplinares para o estudo de desenvolvimento humano.

\section{O que é Desenvolvimento Humano?}

A ciência do desenvolvimento humano propõe princípios ou enunciados, visando estabelecer um acordo acerca do que é entendido como desenvolvimento. Magnusson e Cairns (1996) destacam sete princípios básicos que devem nortear as pesquisas nesta área. O primeiro deles estabelece que a pessoa se desenvolve e funciona psicologicamente como um organismo integrado, em que os elementos de maturação, experiência e cultura se fundem na ontogenia. O segundo ponto enfatiza que a pessoa em desenvolvimento funciona de acordo com uma dinâmica contínua, em um processo de interação com seu ambiente, incluindo as relações com outras pessoas e grupos sociais e culturais. Já, o terceiro destaca que o funcionamento individual depende de influências recíprocas de interação entre subsistemas da própria pessoa, tais como os sistemas cognitivo, emocional, fisiológico, morfológico, conceitual e neurobiológico. Todos esses sistemas agem ao longo do tempo.

Os demais princípios indicam (a) que novos padrões de funcionamento individual surgem durante a ontogenia; (b) que as variações no desenvolvimento produzem diferenças na organização e na configu- ração de funções psicológicas, podendo o desenvolvimento ser acelerado ou retardado; (c) que o funcionamento psicológico é entendido em termos de organização hierárquica a partir de sistemas dinâmicos mais elementares; e, finalmente, (e) que as continuidades e mudanças no desenvolvimento resultam de pressões de forças internas e externas ao organismo.

Portanto, para compreender a complexidade do desenvolvimento humano é necessário adotar uma perspectiva sistêmica que seja capaz de integrar os múltiplos subsistemas do individuo. Isto requer a contribuição de diferentes disciplinas, tais como, a biologia e a psicologia do desenvolvimento, a fisiologia, a neuropsicologia, a psicologia social, a sociologia e a antropologia. Shanahan, Valsiner e Gottlieb (1997) propuseram um conjunto de definições heurísticas, referentes a conceitos de desenvolvimento, que pode ser encontrado paralelamente na maioria dessas disciplinas, facilitando a comunicação multi e interdisciplinar. As definições tratam de estrutura, temporalidade, mudança, padrões de mudança e critérios intelectuais e sociais para distinguir as mudanças associadas ao desenvolvimento.

A respeito da 'estrutura', as disciplinas reconhecem que o desenvolvimento ocorre em um sistema estruturado hierarquicamente, tanto vertical quanto horizontalmente, existindo relações bidirecionais entre eles, isto é, relações de influências mútuas e recíprocas. Na biologia, por exemplo, se assume que a hierarquia vertical do organismo parte do nível mais baixo (fenômenos intra-nucleares), passando por células, tecidos, órgãos, organismos e seu ambiente, envolvendo até outros organismos e o ecossistema físico. A diferenciação horizontal ocorre com base nas interações entre os próprios níveis de cada sistema. Já, na sociologia, a diferenciação vertical abarca desde as díades (ex.: professor-aluno) e grupos pequenos (ex.: sala de aula) até organizações formais (ex.: escolas) e instituições (ex.: sistema educacional), enquanto a diferenciação horizontal considera as conexões entre os níveis contidos em cada sistema. A estrutura pode favorecer ou dificultar os processos de desenvolvimento.

Quanto à ‘temporalidade', há consenso de que as análises em desenvolvimento devem expressar os acontecimentos prévios, ou seja, os eventos em sua seqüência histórica, com o tempo operando em dife- 
rentes níveis dentro do sistema. Cada disciplina utiliza escalas de tempo diferentes e conceitos próprios para abarcar a noção do tempo. Por exemplo, os sociólogos têm estudado a heterocronologia, que se refere ao descompasso do tempo social e do tempo pessoal e às transições que ocorrem antes ou depois do tempo esperado. Segundo Cairns (conforme citado por Shanahan, Valsiner \& Gottlieb, 1997), aspectos filogenéticos podem ser observados no tempo geológico, os aspectos ontogenéticos podem ser estudados na perspectiva do curso de vida e os processos microgenéticos em episódios interativos.

Outra variável que faz parte da definição de desenvolvimento é a 'mudança', que é originada da ruptura da homeostase nos sistemas, seguida por um processo de ajuste que representa a própria mudança (Gottlieb, 2003). As tensões, denominadas de forças de coação, são criadas "no nível estrutural e funcional do organismo, quando este se depara com circunstâncias novas ou adversas à manutenção do seu equilíbrio” (Aspesi, Dessen \& Chagas, no prelo). De acordo com esta noção, o desenvolvimento pode ser produzido (a) por meio de indução, que é a canalização do desenvolvimento em uma direção mais que em outra; (b) de forma facilitadora, promovendo experiências que facilitam ou aceleram estágios consecutivos do processo do desenvolvimento e; (c) por meio de manutenção, em que é mantido o estágio final de um processo de desenvolvimento já alcançado.

Os 'padrões de mudança' indicam que os níveis do sistema são co-acionais, isto é, as mudanças ocorrem em todas as partes dos sistemas envolvidos e estão coordenadas funcionalmente. Assim, a compreensão do desenvolvimento é baseada na noção de que as diferentes estruturas e funções dos sistemas mudam de modo coordenado para adaptar-se ao desequilíbrio da homeostase, para, em seguida, alcançar novo equilíbrio (Shanahan, Valsiner \& Gottlieb, 1997). A última proposição heurística aborda os critérios intelectuais e sociais que definem as mudanças a serem estudadas em desenvolvimento. Tais mudanças são produto de um processo histórico que permite refinar e avaliar as teorias e as evidências empíricas que as fundamentam. Portanto, estudar os processos de desenvolvimento significa estudar mudanças que são perceptíveis ao longo do tempo, mas também os padrões de continuidades, ambos representados nos estágios e transições durante o curso de vida.

Embora o conceito de desenvolvimento esteja ligado à mudança, nem toda mudança é considerada desenvolvimento (Valsiner \& Connolly, 2003). Segundo estes autores, os processos de mudanças, para serem considerados 'desenvolvimento', do ponto de vista científico, requerem a identificação de uma direção a ser seguida ao longo do tempo, embora tal direção não tenha que ser especificada em termos concretos, podendo ser deduzida teoricamente, por exemplo, com o auxílio de recursos estatísticos. No entanto, para detectar uma mudança de desenvolvimento é preciso haver a comparação de dados entre, no mínimo, dois momentos específicos no tempo, determinados em função dos objetivos do estudo.

Outra noção importante, incorporada recentemente ao conceito de desenvolvimento, é a noção de epigênese probabilística do desenvolvimento (Gottlieb, 2003). Em outras palavras, a mudança não é completamente previsível, pois depende das forças de coação recíprocas entre as partes constituintes dos sistemas complexos: genético, neural, comportamental e ambiental, este último subdividido em ambientes físico, social e cultural (Gottlieb, 2003; Shanahan, Valsiner \& Gottlieb, 1997). Por exemplo, os resultados orgânicos ou neurais do desenvolvimento podem ser conseqüência da coação de, pelo menos, dois componentes: pessoa-pessoa, organismo-ambiente, núcleo-citoplasma, dentre outros. De acordo com esses autores, 'experiência' é o termo mais utilizado para referir-se a essas coações e pode ser anatômica, fisiológica e comportamental.

A noção epigenética propõe que o desenvolvimento se caracteriza por um aumento na complexidade da organização em todos os níveis hierárquicos - dos genes, citoplasma, células, órgãos, sistema orgânico, comportamento, até os ambientes social e cultural, e que este aumento em complexidade implica a emergência de novas propriedades funcionais, estruturais e de competências (Gottlieb, 1996). A interdependência entre os diferentes níveis que se influenciam mutuamente, no mínimo de modo bidirecional, leva a abandonar, atualmente, a noção conclusiva da determinação genética das características anatômicas e fisiológicas. Conforme enfatizado por Gottlieb, os experimentos embriológicos têm de- 


\section{Maria Auxiliadora Dessen}

monstrado que as células têm uma multipotencialidade, o que possibilita o seu desenvolvimento em qualquer parte do corpo, de acordo com a interação que mantêm com as outras células ao seu redor e essa tendência se repete em todos os níveis.

Essa noção de que cada aspecto do ambiente poderá ter influências no desenvolvimento com resultados tão diversos, denominada de 'norma de reação’, indica claramente que a pesquisa empírica ainda não tem condições apropriadas para prever as trajetórias dos fenômenos de desenvolvimento. Portanto, as pesquisas contemporâneas precisam incorporar essa noção epigenética e probabilística do desenvolvimento, a fim de obter resultados empíricos compatíveis com os avanços conceituais da ciência do desenvolvimento. A seguir, discute-se a importância dos conceitos de mudanças e continuidades para a compreensão dos fenômenos de desenvolvimento humano e como a abordagem do curso de vida se mostra apropriada para estudar tais fenômenos.

\section{Mudanças e Continuidades no Processo de Desenvolvimento: Dois Conceitos Interligados}

As mudanças existem uma vez que os comportamentos do individuo ocorrem em função de um tempo histórico e de um espaço específico no qual ele está inserido, enquanto que as continuidades existem, na medida em que permanecem os vínculos entre os padrões comportamentais prévios e posteriores (Keller, 1991). A continuidade refere-se à regularidade nos padrões de desenvolvimento, tanto em termos de estrutura e processos, como em termos de mecanismos, o que significa distinguir entre continuidade ao nível de construtos teóricos e continuidade ao nível dos comportamentos observáveis. A continuidade é também vista em termos de padrões previsíveis de relacionamento ou de relações causais entre experiências prévias e posteriores.

Elder (1996) identifica três mecanismos responsáveis pela mudança e continuidade no desenvolvimento humano. O primeiro deles é denominado 'interação continuada' e trata da persistência dos efeitos comportamentais de determinada experiência, que em interação com outras pessoas, tendem a re-criar as mesmas condições. O segundo, a 'ativação situacional', refere-se a situações que são semelhan- tes a experiências significativas vivenciadas pelo indivíduo e que tendem a evocar comportamentos e respostas emocionais similares. Por último, os 'efeitos cumulativos' que tratam da continuidade no comportamento, mantida pela progressiva acumulação de conseqüências do próprio comportamento. Estes mecanismos trazem embutidas as noções de estágio e transição que caracterizam o desenvolvimento humano ao longo do curso de vida.

O estágio é definido como "o conjunto de padrões comportamentais e habilidades características de uma determinada idade ou fase do ciclo de vida do individuo e a transição refere-se aos períodos de passagem de um estágio para outro no ciclo de vida” (Aspesi, Dessen \& Chagas, no prelo). Essas passagens acontecem no marco do ciclo de vida, que é entendido como o conjunto de eventos compreendidos entre o nascimento e a morte e que tem como referência o processo reprodutivo na sociedade (Elder, 1996). As sociedades constroem um conjunto de eventos associados a cada estágio do curso de vida, impondo demandas para as quais a pessoa deve adaptar-se.

Portanto, a continuidade trata dos padrões relacionais e comportamentais que são transferidos entre situações, evocando respostas nas outras pessoas inseridas nos novos contextos de interação, reforçando os padrões iniciais, adaptando-os ao novo contexto. As mudanças no desenvolvimento resultam de acomodações cognitivas e comportamentais que ocorrem de forma simultânea com as mudanças registradas em outros sistemas orgânicos ou ambientais, em determinados momentos do tempo. Isto requer que o desenvolvimento seja analisado em termos sistêmicos, ou seja, considerando o dinamismo entre os diferentes sistemas e tempos de reorganização adaptativa. Uma das abordagens que cumpre tal requisito é a abordagem denominada de 'curso de vida', que incorpora as noções de tempo, de contexto e de processo (Elder, 1996).

A noção de curso de vida implica considerar as mudanças e a interdependência das trajetórias do indivíduo vinculadas à idade que, por sua vez, dependem das mudanças que ocorrem nas sociedades. Por exemplo, a entrada na escola e o momento de aposentar-se variam entre as sociedades. Segundo Elder (1996), para compreender o desenvolvimento, as aná- 
lises deverão incluir evidências do atual funcionamento do ambiente, como estrutura social e processo social, nos seus diferentes níveis: desde o nível mais macro da organização social até grupos menores como escolas e famílias, incluindo estruturas intermediárias e locais como comunidade e vizinhança.

A abordagem do curso da vida considera quatro princípios paradigmáticos: (a) variações geográficas e históricas nas vidas humanas; (b) organização humana e suas restrições sociais moldando as trajetórias do desenvolvimento; (c) o papel central do tempo de oportunidade na estrutura e processo do desenvolvimento; (d) vidas relacionadas ou interdependentes na matriz de relações sociais, no tempo (Elder, 1996). Tais princípios geram, portanto, quatro temas principais a serem estudados: o entrelaçamento das vidas humanas e as mudanças históricas e ambientais, as organizações humanas e as tomadas de decisões e as restrições sociais, o senso de oportunidade das vidas e, por fim, as vidas em relação. Adotar essa abordagem significa focalizar as mudanças nos padrões de vida, levando em conta os seus contextos específicos, enfatizando o papel das mudanças sociais no processo de desenvolvimento do indivíduo.

Conhecer a trajetória do desenvolvimento do indivíduo constitui um desafio para os pesquisadores de diferentes disciplinas, que necessitam, obviamente, de pressupostos comuns para "entenderem como os sistemas múltiplos que influenciam o desenvolvimento individual - dos processos culturais a eventos genéticos e de processos fisiológicos a interações sociais, vão integrando-se no decorrer do tempo, promovendo o funcionamento saudável e adaptativo ou sua conversão" (Magnusson \& Cairns, 1996, p. 9). Portanto, para compreender o desenvolvimento humano, é preciso considerar a emergência e a evolução do indivíduo, em seus diferentes aspectos interligados: biológicos, psicológicos, sociais, culturais e históricos. Aseguir, destaca-se a importância de se considerar a influência dos fatores ambientais no desenvolvimento.

\section{Enfatizando a Inter-Relação Entre Fatores Bi- ológicos e Ambientais no Estudo do Desenvol- vimento}

Seguindo a lógica hierárquica das inter-relações entre os diferentes níveis dos sistemas em de- senvolvimento, uma das tarefas básicas da ciência do desenvolvimento é compreender como o comportamento individual entra em contato com os outros elementos significativos do seu entorno, além de descrever as características desses contatos que são relevantes para o desenvolvimento. De acordo com Magnusson e Cairns (1996), os comportamentos sociais organizam os contatos entre o organismo e o ambiente, promovendo seletivamente os processos de adaptação. Neste sentido, o comportamento passa a ser a interface entre as atividades intra e extra-organismo. Para alguns autores, dentre eles Gariépy (1996), o fato de a integração hierárquica implicar em integração funcional entre os níveis de organização (desde os genes até o ambiente), não significa que se tenha que assumir que o comportamento seja produto dessas interações. Para Gariépy, o comportamento constitui a origem dos processos que permitem a interação entre condições intra e extra-organismo, conduzindo à continuidade e à mudança nos processos adaptativos no decorrer da vida.

Compatível com essa noção, Bronfenbrenner (1992) considera o desenvolvimento humano como "um conjunto de processos por meio dos quais as propriedades do indivíduo e do ambiente interagem e produzem continuidades e mudanças nas características da pessoa e no seu curso de vida” (p. 191). E é pelo processo de desenvolvimento que a pessoa adquire uma concepção mais ampla e diferenciada do meio ambiente ecológico, tornando-se cada vez mais capaz de envolver-se em atividades que revelem suas propriedades, sustentem ou reestruturem o ambiente em níveis de complexidade similares ou maiores, tanto em relação ao conteúdo como à forma (Bronfenbrenner, 1979/1996). Em outras palavras, o desenvolvimento representa uma reorganização contínua dentro da unidade tempo-espaço, que opera no nível das ações, percepções, atividades e interações do indivíduo com o seu mundo, sendo estimulado ou inibido por meio das interações com diferentes participantes do ambiente da pessoa.

Coerente com essas noções, Bronfenbrenner (1977, 1994, 1979/1996, 1999, Bronfenbrenner \& Ceci, 1994; Bronfenbrenner \& Morris, 1998) propõe, então, o ‘Modelo Bioecológico’ para nortear as pesquisas sobre desenvolvimento humano. Esse modelo considera o desenvolvimento como uma acomoda- 


\section{Maria Auxiliadora Dessen}

ção progressiva e mútua entre o organismo humano em desenvolvimento e as mudanças imediatas do ambiente no qual ele vive, durante o curso de vida. Isto requer compreender como o processo de desenvolvimento do indivíduo é afetado pelas relações entre os cenários ou entornos imediatos e contextos sociais mais amplos (formais e informais). Em seu modelo, o ambiente ecológico é concebido em estruturas, cada uma delas contidas em estruturas maiores: o microssistema (relação entre a pessoa em desenvolvimento e o ambiente imediato no qual ela está inserida. Ex: a família, escola, trabalho); o mesossistema (inter-relações entre os cenários principais nos quais o individuo em desenvolvimento participa. Ex: relações entre família e escola); o exossistema (estruturas sociais específicas, formais e informais, que têm efeitos sobre os cenários imediatos da pessoa em desenvolvimento, influindo, delimitando e até determinando o que aí acontece. Ex: as instituições da sociedade que operam em um lugar concreto, tais como a secretaria de educação local); e o macrossistema (protótipos gerais de uma cultura e sub-cultura que estabelecem o padrão das estruturas e atividades em um determinado nível. Ex: sistemas políticos, sociais e econômicos).

A dimensão temporal do desenvolvimento também é considerada no modelo bioecológico, incorporando tanto os eventos históricos, que retratam as mudanças da sociedade na qual o indivíduo está inserido, como também as próprias mudanças do indivíduo ao longo de seu curso de vida. Portanto, o estudo do desenvolvimento humano deve ir além da observação do comportamento de uma ou duas pessoas residindo no mesmo lugar, incluindo os sistemas múltiplos de pessoas interagindo em cenários diferentes, levando em conta os aspectos do ambiente e das situações imediatas (Bronfenbrenner, 1977).

Para lidar com todos estes níveis de análises, simultaneamente, são necessárias descrições e categorizações próprias para o fenômeno em questão, abarcando cada nível da complexidade social. Hinde $(1992,1997)$ propõe três estratégias para lidar com o desafio que representa tratar o desenvolvimento como um todo: (a) enfocar os indivíduos e suas redes sociais distinguindo os níveis de complexidade social, bem como as relações dialéticas entre eles; (b) des- crever, como primeiro passo, as categorias e conceitos com capacidade heurística; e (c) vincular a psicologia do desenvolvimento a outras disciplinas. Hinde, além de enfatizar a importância de as pesquisas considerarem as redes de relacionamento social entre as pessoas, focalizando o indivíduo inserido em seu ambiente, defende a necessidade da contribuição de diversas disciplinas para que os processos de desenvolvimento sejam, de fato, compreendidos.

Pensar o desenvolvimento humano abarcando desde os sistemas fisiológicos e psicológicos, de comportamento individual e de interações entre pessoas, até relações compostas por seqüências de interações entre indivíduos, grupos e sociedades, incluindo o sistema de crenças, valores e mitos e as instituições com seus papéis constituintes, compartilhados pelas pessoas da relação, grupo ou sociedade específica, requer planejamentos de pesquisa orientados por abordagens sistêmicas. Em outras palavras, os estudos deveriam focalizar as relações de trocas entre o organismo em desenvolvimento e o seu ambiente também em desenvolvimento, tendo como pressuposto básico que, nessas trocas, o organismo age sobre o ambiente, de modo a manter tanto uma coerência interna consigo mesmo, quanto com o próprio ambiente, e vice-versa. Deste modo,

\begin{abstract}
"os organismos em desenvolvimento tornamse flexíveis, operando em qualquer nível que seja necessário para encontrar as condições ambientais. A relevância da irreversibilidade do tempo torna-se óbvia aqui: um sistema em desenvolvimento que encontra as condições ambientais X, 'em um dado momento', necessita adaptar-se não somente àquele estado já conhecido das demandas ambientais, mas também em modos que 'antecipem as possíveis mudanças' nestas demandas, em um momento subseqüente.” (Valsiner \& Connolly, 2003, p. 11).
\end{abstract}

Dada a relevância da cultura para o desenvolvimento, o tópico a seguir é dedicado a tecer algumas considerações a respeito da cultura e seu papel no desenvolvimento humano. 


\section{Cultura e Desenvolvimento Humano}

De acordo com Chick (1997), existem, na atualidade, muitas definições de cultura, porém elas podem ser resumidas em quatro categorias: (a) cultura entendida como conhecimentos, crenças, idéias, valores, enfim, algo que está na cabeça das pessoas das diferentes sociedades; (b) cultura entendida como padrões de comportamento distintivos de sociedades particulares; (c) cultura como expressões materiais e comportamentais dos membros de uma sociedade; e (d) cultura como um sistema de informação compartilhada pelo grupo. Independentemente da diversidade de definições sobre o termo, a cultura oferece um sistema de significados compartilhados por meio dos quais a pessoa internaliza valores culturais necessários para o desenvolvimento de competências adaptativas. Mesmo aqueles processos biológicos humanos que são considerados universais (ex: produção hormonal durante a adolescência) podem funcionar de modo bastante diferente, dependendo do contexto cultural no qual a pessoa está inserida (Trommsdorff, 2002).

Super e Harkness (1999) destacam que o elemento mais importante da cultura em relação ao desenvolvimento humano é o fato de ela constituir-se em uma realidade imediata, presente e compartilhada pelos membros de uma comunidade, possuindo potencial para permear todas as experiências e comportamentos das pessoas. Para esses autores, as pessoas constroem o ambiente por meio dos costumes, dos comportamentos, das situações, das crenças e dos valores, indicando que um determinado ambiente é formado por uma estrutura sistemática estática e ao mesmo tempo dinâmica. Estrutura estática refere-se às características típicas do ambiente imediato da pessoa (ex: família), em uma dada cultura ou sub-cultura, que repercutem diretamente no seu desenvolvimento, tais como a natureza das interações verbais e a distribuição de tarefas de cuidado no âmbito da família. E são essas regularidades ao longo do tempo que dão continuidade à experiência do desenvolvimento. Por outro lado, a estrutura dinâmica do ambiente humano refere-se às respostas diferenciais entre as pessoas, segundo as características próprias de idade, temperamento, sexo, origem racial etc. e que são responsáveis pela ‘co-variância reativa' entre pessoa e ambiente. Focalizar essa estrutura como unidade de análise em pesquisa implica observar diferenças individuais entre grupos e entre culturas.

Existem variações individuais dentro de uma mesma cultura e, também, variações de padrões de comportamento entre os membros de diferentes culturas. Essas diferenças resultantes da exposição a fatores culturais distintos são perceptíveis em todos os aspectos do desenvolvimento humano, desde a cognição até o comportamento social, por exemplo. A comparação do funcionamento psicológico em contextos culturais distintos, uma das tarefas da psicologia transcultural, possibilita testar pressupostos universais acerca do comportamento humano e das relações sociais, ressaltando, assim, o papel de culturas específicas nesses processos, resultando em dados valiosos para os estudos sobre desenvolvimento humano (Trommsdorff, 2002).

Mas, não se pode deixar de considerar, dentro do ambiente, os aspectos subjetivos que, sem serem necessariamente tangíveis, têm a capacidade de mudar as trajetórias do desenvolvimento do indivíduo e do ambiente, ao longo do tempo. Alguns exemplos destes aspectos subjetivos são o código de comunicação que o indivíduo estabelece para entrar em contato com o seu meio, os símbolos que medeiam o seu comportamento e as regras implícitas e explicitas que organizam as interações entre as pessoas. Estes elementos subjetivos do ambiente são basicamente estímulos sociais que, ao entrarem em contato com o indivíduo, dão forma à bagagem psicológica.

Como é que os elementos da cultura se integram de forma a influenciar diretamente o desenvolvimento humano, particularmente nas primeiras fases do curso de vida? Acerca da organização cultural do desenvolvimento, Super e Harkness (1999) propõem o conceito de 'nicho do desenvolvimento', composto por: (a) contexto físico-social da vida diária da criança, entendido como os objetos e pessoas que circundam a criança, e que representam riscos e apoios para o seu crescimento, e os tipos de interações que eventualmente ocorrem entre eles; (b) costumes regulados culturalmente sobre o cuidado e educação da criança, incluindo as práticas cotidianas, úteis e significativas, que são aplicadas inconscientemente e que parecem ser óbvias e naturais; e (c) a psicologia 


\section{Maria Auxiliadora Dessen}

dos cuidadores, que reflete o sistema de crenças culturalmente organizado acerca do comportamento e desenvolvimento da criança ('etnoteorias parentais'). Todos esses componentes do nicho de desenvolvimento merecem atenção dos pesquisadores da área de desenvolvimento humano, embora, o primeiro deles seja de particular importância, uma vez que o contexto físico e social delimita as oportunidades de desenvolvimento da pessoa.

Não basta estudar os 'nichos de desenvolvimento'; é preciso, também, testar as teorias de desenvolvimento em ambientes culturais distintos para evitar vieses provenientes da produção de conhecimento limitada a populações de países ocidentais industrializados. Tais vieses eurocêntricos e etnocêntricos, característicos das teorias contemporâneas, contradizem os princípios sistêmicos do desenvolvimento humano (Dasen \& Mishra, 2000; van de Vijver \& Leung, 2000).

\section{Considerações Finais}

Neste artigo, argumenta-se que o desenvolvimento precisa ser estudado considerando desde as unidades biológicas até as estruturas sociais, tendo como interface os padrões comportamentais. Isto requer a adoção de um conceito de desenvolvimento que reflita os avanços ocorridos nas últimas décadas, em diferentes disciplinas dedicadas ao estudo desta variável, como também a adoção de modelos sistêmicos. Segundo Lawrence e Dodds (1997), o desenvolvimento deve ser visto como "uma estrutura sistêmica de relações bidirecionais entre níveis verticais e horizontais, ocorrendo em um tempo social e pessoal, sendo as mudanças probabilísticas e manifestadas em padrões de coações coordenadas por meio de níveis do funcionamento humano” (p. 293). Portanto, é necessário desenvolver planejamentos de pesquisa que sejam consistentes com a noção de desenvolvimento constituído por mudanças que ocorrem em função do tempo e das características do contexto, com as interações entre sistemas internos e externos ao indivíduo, provocando o surgimento de novos padrões de funcionamento.

Para estudar o desenvolvimento de forma integrada, é necessário reconhecer que sua complexidade implica o envolvimento de diferentes disciplinas científicas, dentre as quais a biologia, a psicologia, a fisiologia, a neuropsicologia, a sociologia e a antropologia. No entanto, a comunicação multi e interdisciplinar precisa ser cuidadosa. Lawrence e Dodds (1997) explicam, por exemplo, que a simples transposição de conceitos de uma disciplina para outra não é adequada, pois o uso de conceitos equivalentes entre disciplinas deve ser antecedido de uma análise cuidadosa do termo, para cada uma delas, e pela verificação do suficiente paralelismo desses conceitos entre as diferentes áreas. Nesse sentido, acredita-se que os princípios e os conceitos básicos sobre os processos de desenvolvimento propostos pela ciência do desenvolvimento humano facilitam a comunicação entre as disciplinas, provendo uma linguagem comum para a discussão dos dados empíricos.

A ciência do desenvolvimento tem o desafio de entender como os sistemas múltiplos influenciam o desenvolvimento individual, isto é, como os processos culturais e os eventos genéticos e fisiológicos se integram ao longo do curso de vida, promovendo o funcionamento saudável e adaptativo da pessoa (Magnusson \& Cairns, 1996). Portanto, os estudos em desenvolvimento precisam analisar a integração ontogenética considerando o contexto e o tempo. As mudanças temporais são utilizadas para esclarecer os mecanismos do desenvolvimento e demonstram sua operação em instâncias concretas de adaptação. Por exemplo, vínculos mediadores entre a ontogenia e os estudos temporais podem ser vistos em análises detalhadas dos processos de transmissão e de mudanças entre gerações (The Carolina Consortium on Human Development, 1996).

Embora os conceitos e pressupostos da ciência do desenvolvimento humano tenham sido sistematizados na última década do século $\mathrm{XX}$, a sua contribuição tem sido notória, particularmente no que tange às integrações conceituais e à proposição de estratégias metodológicas e analíticas para abordar os fenômenos do desenvolvimento humano (Dessen, no prelo). Tais avanços precisam, agora, ser compartilhados entre os pesquisadores de desenvolvimento, visando à implementação de projetos de pesquisa que tragam contribuições relevantes para a compreensão dos processos de desenvolvimento humano neste século que se inicia. 


\section{Referências Bibliográficas}

Aspesi, C., Dessen, M.A. \& Chagas, J. (no prelo). A ciência do desenvolvimento humano: uma perspectiva interdisciplinar. Em M.A. Dessen \& A.L. Costa-Junior (Orgs.), A ciência do desenvolvimento humano: tendências atuais $e$ perspectivas futuras. Porto Alegre: Artmed Editora S.A.

Bronfenbrenner, U. (1977). Toward an experimental ecology of human development. American Psychologist, 32, 513-531.

Bronfenbrenner, U. (1992). Ecological systems theory. Em R. Vasta (Org.), Six theories of child development (pp. 187-249). London: Jessica Kingsley.

Bronfenbrenner, U. (1994). Ecological models of human development. Em T. Husten \& T.N. Postelethwaite (Orgs.), International Encyclopedia of Education (2a. ed., Vol 3, pp. 16431647). New York: Elsevier Science.

Bronfenbrenner, U. (1996). A ecologia do desenvolvimento humano: experimentos naturais $e$ planejados. (M.A.Veríssimo, Trad.) Porto Alegre: Artes Médicas. (Trabalho originalmente publicado em 1979)

Bronfenbrenner, U. (1999). Environments in developmental perspective: Theoretical and operational models. Em S.L. Friedman \& T.D. Wachs (Orgs.), Measuring environment across the life span: Emerging methods and concepts (pp. 3-28). Washington, DC: American Psychological Association.

Bronfenbrenner, U. \& Ceci, S. (1994). Nature-nurture reconceptualized in developmental perspective: A bioecological model. Psychological Review, 101, 568-586.

Bronfenbrenner, U. \& Morris, P. A. (1998). The ecology of developmental process. Em W. Damon \& R.M. Lerner (Orgs.), Handbook of child psychology: Vol. 1. Theoretical models of human development (5a. ed., pp. 993-1028). New York: Wiley.
Chick, G. (1997). Culture complexity: The concept and its measurement. Cross-Cultural Research, 31, 275-307.

Dasen, P.R. \& Mishra, R.C. (2000). Cross-cultural views on human development in the third millennium. International Journal of Behavioral Development, 24, 428-434.

Dessen, M.A. (no prelo). Construindo uma ciência do desenvolvimento humano: passado, presente e futuro. Em M.A. Dessen \& A.L. Costa-Junior (Orgs.), A ciência do desenvolvimento humano: tendências atuais e perspectivas futuras. Porto Alegre: Artmed Editora S.A.

Elder, G. (1996). Human lives in changing societies: Life course and developmental insights. Em R.B. Cairns, G.H. Elder \& E.J. Costello (Orgs.), Developmental science (pp. 31-62). New York: Cambridge University Press.

Gariépy, J.L. (1996). The question of continuity and change in development. Em R.B. Cairns, G.H, Elder \& E.J. Costello (Orgs.), Developmental science (pp. 78-96). New York: Cambridge University Press.

Gottlieb, G. (1996). Developmental psychobiological theory. Em R.B. Cairns, G.H, Elder \& E.J. Costello (Orgs.), Developmental science (pp. 63-77). New York: Cambridge University Press.

Gottlieb, G. (2003). Probabilistic epigenesis of development. Em J. Valsiner \& K. Connolly (Orgs.), Handbook of developmental psychology (pp. 3-17). London: Sage Publications.

Hinde, R.A. (1992). Developmental psychology in the context of other behavioral sciences. Developmental Psychology, 28, 1018-1029.

Hinde, R.A. (1997). Relationships: A dialetical perspective. London: Psychology Press.

Keller, H. (1991). A perspective on continuity in infant development. Em M.E. Lamb \& H. Keller (Orgs.), Infant development: Perspectives form German-speaking countries (135-150). Hillsdale, NJ: Lawrence Erlbaum. 


\section{Maria Auxiliadora Dessen}

Lawrence, J.A. \& Dodds, A.E. (1997). Conceptual transposition, parallelism, and interdisciplinary communication. Em J. Tudge, M.J. Shanahan \& J. Valsiner (Orgs.), Comparisons in human development: Understanding time and context (pp. 293-303). New York: Cambridge University Press.

Magnusson, D. \& Cairns, R. (1996). Developmental science: Toward a unified framework. Em R.B. Cairns, G.H. Elder \& E.J. Costello (Orgs.), Developmental science (pp. 7-30). New York: Cambridge University Press.

Shanahan, M.J., Valsiner, J. \& Gottlieb, G. (1997). Developmental concepts across disciplines. Em J. Tudge, M.J. Shanahan \& J. Valsiner (Orgs.), Comparisons in human development: Understanding time and context (pp. 34-69). New York: Cambridge University Press.

Super, C.M. \& Harkness, S. (1999). The environment as culture in developmental research. Em S.L. Friedman \& T.D. Wachs (Orgs.), Measuring environment across the life span: Emerging methods and concepts (pp. 279-323). Washington, DC: American Psychological Association

The Carolina Consortium on Human Development (1996). Development science: A collaborative statement. Em R.B. Cairns, G.H. Elder \& E.J. Costello (Orgs.), Developmental science (pp. 1-6). New York: Cambridge University Press.

Trommsdorff, G. (2002). An eco-cultural and interpersonal relations approach to development of the lifespan. Em W.J. Lonner, D.L. Dinnel, S.A. Hayes \& D.N. Sattler (Orgs.), Online Readings in Psychology and Culture (Unit 12, Chapter 1), (http://www.wwu.edu/ culture), Center for Cross-Cultural Research, Western Washington University, Bellingham, Washington USA.

Valsiner, J. \& Connolly, K. (2003). The nature of development: The continuing dialogue of processes and outcomes. Em J. Valsiner \& K. Connolly (Orgs.), Handbook of developmental psychology (pp. 9-18). London: Sage Publications. van de Vijver, F. \& Leung, K. (2000). Methodological issues in psychological research on culture. Journal of Cross-Cultural Psychology, 31, 33-51.

Apoio CNPq - primeira autora, apoio Universidade de Sonora, México, segunda autora. 